\title{
Prospecção científica e tecnológica: uso de nanopartículas de goma do cajueiro, avaliação das atividades anticolinesterásicas $e$ antioxidantes da espécie vegetal barbacenia blanchetii para aplicação na terapia da doença de Alzheimer
}

A nanotecnologia é uma ciência que tem ganhado espaço nos últimos anos, principalmente na área médica, farmacêutica e química, pois esta ciência permite a manipulação da matéria na

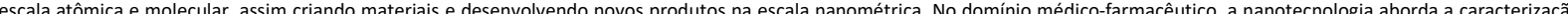

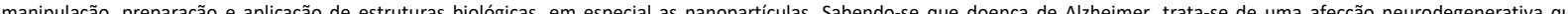
acomete cerca de 35,6 milhões de pessoas no mundo todo sendo na maioria idosos, os medicamentos anticolinesterásicos são os mais indicados para o tratamento desta doença, existindo atualmente no mercado 5 fármacos aprovados pela Food and Drug Administration (FDA) para a comercialização e tratamento da doença de Alzheimer, com excecão da memantina. As outras 4 drogas permitidas no tratamento da DA se baseiam na atividade anticolinesterásica, sendo que dessas 4, 2 desses fármacos, a galantamina (Reminyl, Razadyne) e a rivastigmina (Exelon) são de origem natural. No contexto fitoquímico, a espécie vegetal Barbacenia blanchetii que ocorre nos campos rupestres da Chapada Diamantina no Estado brasileiro da Bahia, rica em flavonoides, onde estudos realizados com esta planta mostraram resultados consideráveis frente à ação anticolinesterásica, principalmente sob a enzima butirilcolinesterase. Diante dessas informações, investigou-se se há o uso de extratos incorporados em nanopartículas, obtidas a partir da goma do cajueiro, e também do óleo essencial extraído dessa espécie vegetal, a Barbacenia blanchetii e suas aplicações na terapia da doença de Alzheimer, por meio de uma prospecção tecnológica nas principais bases de patentes e periódicos, onde não foram encontrados documentos de patentes relacionados a esse vegetal e apenas 1 artigo disponível nos periódicos PubMed e Web of Science.

Palavras-chave: Barbacenia blanchetii; Nanopartícula; Goma do Cajueiro; Doença de Alzheimer; Prospecção.

\section{Scientific and technological prospection: use of cashew gum nanoparticles, evaluation of anticolinesterastic and antioxidant activities of the vegetable species barbacenia blanchetii for application in the therapy of Alzheimer disease}

Nanotechnology is a science that has gained interest in recent years, especially in the medical, pharmaceutical and chemical fields, because this science allows the manipulation of matter at the atomic and molecular scale, creating new materials and developing new products at the nanoscale. In the medical pharmaceutical field, nanotechnology deals with the characterization, manipulation, preparation and application of biogis structures, especially nonoparticles. It is known that Alzheimer's disease is a neurodegenerative disorder that approved by the Food and Drug Administration (FDA) for the commercialization and treatment of Alzheimer's disease, with the exception of memantine. The other 4 drugs allowed in the treatment of $A D$ are based on anticholinesterase activity, and of these 4,2 of these drugs, galantamine (Reminyl, Razadyne) and rivastigmine (Exelon) are naturally occurring. In the phytochemical context, the Barbacenia blanchetii plant species that occurs in the Chapada Diamantina rupestrian fields in the brazilian State of Bahia, is rich in flavonoids, where studies with this plant showed considerable results against the anticholinesterase in the Chapada Diamantina rupestrian fields in the brazilian State of Bahia, is rich in flavonoids, where studie extracts incorporated in nanoparticles, obtained from cashew gum, and also of essential oil extracted from this plant species, Barbacenia blanchetii and it's applications in the therapy of Alzheimer's disease, by means of a technological survey in the main patent and periodical bases, where no patent documents related to this vegetable were found and only one article available in the periodicals PubMed and Web of Science.

Keywords: Barbacenia blanchetii; Nanoparticles; Cashew gum; Alzheimer's disease; Prospection.

Topic: Farmacologia

Reviewed anonymously in the process of blind peer

Viviane Lima Silva (ic)

Centro Universitário Brasileiro de Tecnologia Avançada, Brasil

http://lattes.cnpq.br/9968454356827398

http://orcid.org/0000-0002-5584-4185

viviannelyma956@gmail.com
Received: 10/02/2021

Approved: 20/03/2021
Referencing this:

SILVA, V. L.. Prospecção científica e tecnológica: uso de nanopartículas de goma do cajueiro, avaliação das atividades anticolinesterásicas e antioxidantes da espécie vegetal barbacenia blanchetii para aplicação na terapia da doença de alzheimer. Scire Salutis, v.11, n.2, p.14-24, 2021. DOI: http://doi.org/10.6008/CBPC2236-9600.2021.002.0002 
Prospeç̧ão científica e tecnológica: uso de nanopartículas de goma do cajueiro, avaliação das atividades anticolinesterásicas e antioxidantes da

\section{INTRODUÇÃO}

A doença de Alzheimer (DA) foi descoberta em 1907 e se enquadra como uma afecção multifatorial, pois as suas causas ainda não foram elucidadas, de cunho neurodegenerativa irreversível e progressiva, a qual se apresenta geralmente em torno dos 60 anos de idade, mas pode também desenvolver-se precocemente, em torno dos 40 anos, ligado ao número de casos na quantidade de incidências da doença na família, e é um dos grandes problemas de demência que mais acomete a população mundial afetando mais de 35,6 milhões de pessoas na maioria idosos, pois essa doença traz para o acometido muitos distúrbios cognitivos, como por exemplo, a perda de memória, dificuldades no uso ou a perda da linguagem, perda da razão, da habilidade de cuidar-se, descontrole emocional, comportamental e motivacional, enquadrando-se também como sintomas da doença a perda da capacidade motora, quadros de depressão e delírios, o que induz o indivíduo a incapacidade social e mental, impondo a sociedade e a família uma enorme carga emocional e financeira por meio da prestação de cuidados e perdas salariais para o tratamento da doença (HOLMES, 2012; KONRATH et al., 2013; SINGH et al., 2013; RIEDEL, 2014; SCOTTI et al., 2014).

Acredita-se que o principal fator de risco para o desenvolvimento desta doença é o avanço da idade, onde $25 \%$ a $30 \%$ da população mundial é acometida por demência, e que se novos e mais eficientes tratamentos não forem encontrados prevê-se que em 2030 o número de ocorrências de demências dobre, ou seja, chegue a quase 70 milhões, sendo em torno de 7,7 milhões o número total de ocorrência de demências ao ano, o que implica no aparecimento de um novo caso a cada 4 segundos, onde dentre os tipos de demência mais comuns, a doença de Alzheimer, representa cerca de $70 \%$ a $75 \%$ dos casos de demência acima da faixa etária de 65 anos (OTAEGUI-ARRAZOLA et al., 2014).

Acredita-se que a progressão da doença ocorra em três estágios principais, sendo no estágio inicial, que pode durar em torno de 2 a 4 anos, o sistema colinérgico é danificado por meio de danos seletivos aos neurônios relacionados à memória e aos neurônios específicos do sistema límbico, ou hipocampo, onde há o encolhimento do mesmo em cerca de $25 \%$ do seu volume inicial, prevalecendo sintomas típicos como a perda leve da memória, principalmente as relacionadas as informações recentemente aprendidas e pequenas dificuldades de comunicação, já no estágio intermediário que pode durar cerca de 2 a 10 anos, o indivíduo com DA apresenta reflexo de devastação do sistema límbico e do hipocampo, declínio nos níveis de acetilcolina, um neurotransmissor relacionado com a retenção da memória e a aprendizagem, os sintomas apresentados nos indivíduos com DA neste estágio são a dificuldade para lembrar fatos que remetem ao próprio passado, as alterações na personalidade como raiva, confusão, tristeza, falta de concentração e orientação, no último estágio da DA, o estágio avançado, com duração média de 3 anos, o doente apresenta lesões de grande parte do sistema límbico e ao ataque dos neurônios colinérgicos devido a deposição do peptídeo $\beta$-amiloide, culminando com a morte do paciente (MORZELLE, 2012).

Alguns autores explicam que diversos mecanismos têm sido propostos como possíveis explicações para o aparecimento da doença de Alzheimer, como o depósito de peptídeos amiloides em placas que 
Prospeç̧ão científica e tecnológica: uso de nanopartículas de goma do cajueiro, avaliação das atividades anticolinesterásicas e antioxidantes da

causam a destruição de neurônios por desenvolver um processo inflamatório crônico nas regiões afetadas, além de alterar a regulação do cálcio que é essencial para a condução dos estímulos nervosos, e aumentar a produção de radicais livres que são tóxicos para as células nervosas, outros mecanismos descritos também para o surgimento da DA são a hiperfosforilação da proteína tau, disfunção colinérgica, estresse oxidativo, disfunção sináptica, inflamação consequente ao acúmulo de peptídeos $\beta$-amiloide (DERMARIN, 2011; MAHDY et al., 2012; POHANKA, 2014; SCOTTI et al., 2014).

O mal de Alzheimer, apesar de ter sido descoberto a mais de um século pelo médico Alois Alzheimer, ainda não tem cura, então neste contexto, para pesquisas em se tratando da terapia da doença de Alzheimer segue-se três abordagens terapêuticas investigadas que são o restabelecimento dos níveis de neurotransmissores, o uso de antioxidantes, e a utilização de agentes capazes de interferir em aspectos mais específicos da doença como a cascata amiloide e proteína tau, sendo o uso de inibidores de AChE o método mais bem estabelecido no tratamento da DA (KONRATH et al., 2013; RAMIREZ-BERMUDEZ, 2012).

Atualmente existem no mercado cinco fármacos aprovados pela Food and Drug Administration (FDA) para a comercialização e tratamento da doença de Alzheimer, com exceção da memantina, as outras quatro drogas se baseiam na atividade anticolinesterásica, sendo que dessas quatro, dois desses fármacos, a galantamina (Reminyl, Razadyne) e a rivastigmina (Exelon) são de origem natural (MURRAY et al., 2013).

A galantamina, comercializada com os nomes de Reminyl e Razadyne, trata-se de um alcaloide isolado de plantas da família Amaryllidaceae, onde apresenta uma longa ação seletiva, reversível, pois a forma da enzima é sempre regenerada, e competitiva para inibir a AChE, sendo um dos medicamentos mais efetivos no tratamento da DA pelo fato de apresentar poucas limitações, já a Rivastigmina comercializada com o nome de Exelon, é um dos medicamentos mais utilizados no tratamento da doença de Alzheimer, sendo capaz de inibir tanto a enzima acetilcolinesterase quanto a butirilcolinesterase, apresentando assim, uma maior eficácia quanto ao aumento dos níveis cerebrais de acetilcolina (SEIDL, 2010; MURRAY et al., 2013).

Apesar do uso dos medicamentos acima como alternativa terapêutica no tratamento da doença de Alzheimer, os mesmos trazem alguns efeitos colaterais, como a rivastigmina que apresenta efeitos gastrointestinais adversos, associados ao aumento de peso dos pacientes, a Tacrina, comercializada com o nome de Cognex, mesmo sendo o primeiro inibidor reversível da AChE apresentou hepatoxicidade, levando ao aumento das transminases hepáticas, resultando em hepatite medicamentosa, o que provocou a retirada do medicamento do mercado (SEIDL, 2010; MURRAY et al., 2013). Dentro desse contexto seria viável o uso de medicamentos

Em virtude de sua origem natural e complexa estrutura nitrogenada, os alcaloides são considerados os candidatos mais promissores no tratamento da doença de Alzheimer, mas além destes, diferente das drogas a base de alcaloides utilizadas no tratamento da DA, os compostos não alcaloides, como por exemplo, os flavonoides, apresentam atividade antioxidante, anti-inflamatória, antimicrobiana e antitumoral, destacando-se dentre estas atividades a inibição enzimática das colinesterases, o que tornam compostos não alcaloides, como os flavonoides, grandes candidatos e promissores no desenvolvimento de 
Prospeç̧ão científica e tecnológica: uso de nanopartículas de goma do cajueiro, avaliação das atividades anticolinesterásicas e antioxidantes da

fármacos para a aplicação no tratamento da doença de Alzheimer (PEREIRA et al., 2010; KONRATH et al., 2013; MURRAY et al., 2013).

Nos últimos anos, diversas revisões a respeito de novos inibidores de AChE obtidos de plantas, fungos e organismos marinhos tem sido publicados, sendo a maioria desses inibidores pertencentes ao grupo dos alcaloides, entretanto, diversos compostos não alcaloides com potente atividade anticolinesterásica também foram obtidos a partir de fontes naturais, como por exemplo, os terpenos, as xantonas, cumarinas, flavonoides e outros compostos fenólicos, sendo que os extratos obtidos de plantas tem demonstrado serem excelentes veículos carreadores de substâncias bioativas capazes não apenas de inibir a atividade anticolinesterásica, mas também de exercer funções antioxidantes e anti-inflamatória (KONRATH et al., 2013; MURRAY et al., 2013).

Diante dos expostos acima, a busca por novos inibidores de colinesterases, de origem vegetal, constitui uma alternativa viável e interessante na tentativa de desenvolver compostos com maior eficácia para o tratamento da DA, pelo fato de apresentar poucas limitações, já que um dos medicamentos mais eficazes no tratamento da DA, a galantamina, é um produto natural e apresenta menos efeitos colaterais que outros inibidores utilizados, além das substâncias antioxidantes que são citadas como forma de prevenir e como adjuvante ao tratamento desta doença, por promover neuroproteção, o que evita dano oxidativo ao tecido neuronal, inibindo assim, a formação de placas do peptídeo amiloide, uma das características da Doença de Alzheimer (FEITOSA, 2015).

A AChE encontra-se em maior abundância no cérebro humano, sendo por muitas vezes esquecido o papel da BuChE (butirilcolinesterase), pois acredita-se que a BuChE presente no cérebro desempenhe papel adicional importante na transmissão colinérgica e também exerça influência na expressão da enzima AChE. Trabalhos anteriores com os extratos hexânico, acetato de etila e metanólico, ricos em flavonoides, da espécie Barbacenia blanchetii inibiram, respectivamente, a butirilcolinesterase em concentração dependente em $62,33 \%, 67,89 \%$ e 70,92\% apresentando assim atividade anticolinesterásica considerável frente à enzima butirilcolinesterase (BARBOSA, 2012).

Diante destas informações, torna-se notável a relevância e a viabilidade da Barbacenia blanchetii como espécie vegetal promissora para o tratamento da doença de Alzheimer, sendo necessária a realização de uma prospecção científica e tecnológica com o intuito de se investigar o estado atual da arte e da técnica relacionados a esse vegetal baseando-se no número de publicações e patentes depositadas.

\section{METODOLOGIA}

Para a realização da prospecção tecnológica foram analisadas as patentes depositadas nos seguintes bancos de dados: European Patent Office (EPO), World Intellectual Property Organization (WIPO), United States Patent and Trademark Office (USPTO) e no Instituto Nacional de Propriedade Industrial (INPI), bem como nas principais bases de dados de publicação de periódicos: PubMed, SciELO, Web of Science e Science Direct.

Para a efetivação das buscas, na base de dados de periódicos e patentes internacionais, foram 
Prospecção científica e tecnológica: uso de nanopartículas de goma do cajueiro, avaliação das atividades anticolinesterásicas e antioxidantes da espécie vegetal barbacenia blanchetii para aplicação na terapia da doença de Alzheimer

SILVA, V. L.

inseridas as palavras-chave combinadas no idioma inglês: "Barbacenia", "Barbacenia blanchetii", "Barbacenia blanchetii AND nanothechology", "Barbacenia blanchetii AND nanoparticle", "Barbacenia blanchetii AND nanoparticle cashew gum", "Barbacenia blanchetii AND essential oil", "Barbacenia blanchetii AND aqueous extract", "Barbacenia blanchetii AND hydroalcoholic extract", "Barbacenia blanchetii AND alcohol extract", "Barbacenia blanchetii AND ethanolic extract", "Barbacenia blanchetii AND ethyl acetate extract", "Barbacenia blanchetii AND hexane extract", "Barbacenia blanchetii AND methanolic extract", "Barbacenia blanchetii AND antioxidant activity", "Barbacenia blanchetii AND Alzheimer's disease".

Para a realização da busca de documentos na base nacional de dados, no INPI, a combinação de palavras utilizadas em português, foram as seguintes: Barbacenia, Barbacenia blanchetii, Barbacenia blanchetii e nanotecnologia, Barbacenia blanchetii e nanopartícula, Barbacenia blanchetii e nanopartícula de goma do cajueiro, Barbacenia blanchetii e óleo essencial, Barbacenia blanchetii e extrato aquoso, Barbacenia blanchetii e extrato hidroalcoólico, Barbacenia blanchetii e extrato alcoólico, Barbacenia blanchetii e extrato etanólico, Barbacenia blanchetii e extrato de acetato de etila, Barbacenia blanchetii e extrato hexânico, Barbacenia blanchetii e extrato metanólico, Barbacenia blanchetii e atividade antioxidante, Barbacenia blanchetii e doença de Alzheimer. O levantamento dos dados foram realizados no período de 2 de maio de 2018 a 27/06/2018 e os gráficos e tabelas foram gerados no programa Microsoft Excel 2010.

\section{DISCUSSÃO TEÓRICA}

As pesquisas listadas na tabela 1 foram realizadas com a inserção das palavras-chave, as quais foram cruzadas no intuito de se obter um parâmetro maior relacionado ao uso da espécie vegetal Barbacenia Blanchetii.

Tabela 1: Depósitos de patentes relacionados por palavras-chave distribuídos nas bases de dados.

\begin{tabular}{|c|c|c|c|c|}
\hline PALAVRAS-CHAVE & INPI & EPO & WIPO & USPTO \\
\hline Barbacenia & 0 & 0 & 0 & 0 \\
\hline Barbacenia blanchetii & 0 & 0 & 0 & 0 \\
\hline Barbacenia blanchetii AND nanothechology & 0 & 0 & 0 & 0 \\
\hline Barbacenia blanchetii AND nanoparticle & 0 & 0 & 0 & 0 \\
\hline Barbacenia blanchetii AND nanoparticle cashew gum & 0 & 0 & 0 & 0 \\
\hline Barbacenia blanchetii AND essential oil & 0 & 0 & 0 & 0 \\
\hline Barbacenia blanchetii AND aqueous extract & 0 & 0 & 0 & 0 \\
\hline Barbacenia blanchetii AND hydroalcoholic extract & 0 & 0 & 0 & 0 \\
\hline Barbacenia blanchetii AND alcohol extract & 0 & 0 & 0 & 0 \\
\hline Barbacenia blanchetii AND ethanolic extract & 0 & 0 & 0 & 0 \\
\hline Barbacenia blanchetii AND ethyl acetate extract & 0 & 0 & 0 & 0 \\
\hline Barbacenia blanchetii AND hexane extract & 0 & 0 & 0 & 0 \\
\hline Barbacenia blanchetii AND methanolic extract & 0 & 0 & 0 & 0 \\
\hline Barbacenia blanchetii AND antioxidant activity & 0 & 0 & 0 & 0 \\
\hline Barbacenia blanchetii AND anticholinesterase activity & 0 & 0 & 0 & 0 \\
\hline Barbacenia blanchetii AND Alzheimer's disease & 0 & 0 & 0 & 0 \\
\hline
\end{tabular}

De acordo com os resultados encontrados, propostos na tabela 1, percebe-se a inexistência de patentes depositadas. Para a construção da tabela 2, foram inseridas as palavras-chave relacionadas na 
Prospeç̧ão científica e tecnológica: uso de nanopartículas de goma do cajueiro, avaliação das atividades anticolinesterásicas e antioxidantes da

tabela 1, bem como, as mesmas traduzidas para a língua portuguesa, filtrando-se a busca por área de interesse, a qual também apontou nenhum depósito de patente nos referidos bancos de dados.

Tabela 2: Quantitativo do depósito de patentes por áreas de utilização da espécie Barbacenia Blanchetii.

\begin{tabular}{|l|c|c|}
\hline ÁREAS DE INTERESSE & REGISTROS & $\%$ \\
\hline Agricultura & 0 & 0 \\
\hline Bioquímica e Biologia Molecular & 0 & 0 \\
\hline Ciência Ambiental & 0 & 0 \\
\hline Farmacologia & 0 & 0 \\
\hline Fitotecnia & 0 & 0 \\
\hline Medicina & 0 & 0 \\
\hline Química & 0 & 0 \\
\hline Tecnologia de Alimentos & 0 & 0 \\
\hline Outras & 0 & \\
\hline
\end{tabular}

Na tabela 3 propõem-se os resultados obtidos por meio das buscas relacionadas à produção científica, disponível em periódicos, relativa ao uso da espécie vegetal Barbacenia blanchetii e também de pesquisas ligadas ao gênero Barbacenia.

Tabela 3: Número de artigos científicos publicados relacionados às palavras-chave.

\begin{tabular}{|c|c|c|c|}
\hline PALAVRAS-CHAVE & PubMed & SCIELO & Science Direct \\
\hline Barbacenia & 6 & 0 & 20 \\
\hline Barbacenia blanchetii & 1 & 0 & 0 \\
\hline Barbacenia blanchetii AND nanothechology & 0 & 0 & 0 \\
\hline Barbacenia blanchetii AND nanoparticle & 0 & 0 & 0 \\
\hline Barbacenia blanchetii AND nanoparticle cashew gum & 0 & 0 & 0 \\
\hline Barbacenia blanchetii AND essential oil & 0 & 0 & 0 \\
\hline Barbacenia blanchetii AND aqueous extract & 0 & 0 & 0 \\
\hline Barbacenia blanchetii AND hydroalcoholic extract & 0 & 0 & 0 \\
\hline Barbacenia blanchetii AND alcohol extract & 0 & 0 & 0 \\
\hline Barbacenia blanchetii AND ethanolic extract & 0 & 0 & 0 \\
\hline Barbacenia blanchetii AND ethyl acetate extract & 1 & 0 & 0 \\
\hline Barbacenia blanchetii AND hexane extract & 1 & 0 & 0 \\
\hline Barbacenia blanchetii AND methanolic extract & 1 & 0 & 0 \\
\hline Barbacenia blanchetii AND antioxidant activity & 0 & 0 & 0 \\
\hline Barbacenia blanchetii AND anticholinesterase activity & 1 & 0 & 0 \\
\hline Barbacenia blanchetii AND Alzheimer's disease & 0 & 0 & 0 \\
\hline
\end{tabular}

De acordo com a tabela 3, na base de dados PubMed foram encontrados seis artigos publicados, sendo que dois artigos dessa base encontram-se também publicados na base de dados Web of Science, os quais faziam relação a palavra-chave Barbacenia, sendo que apenas um, dentre esses artigos, de autoria de Barbosa et al. (2015) intitulado "RP-HPLC-DAD-MS(n) Analysis and Butyrylcholinesterase Inhibitory Activity of Barbacenia blanchetii Extracts", fazia alusão a espécie vegetal Barbacenia blanchetii, o qual apresentava estudos relativos a atividade anticolinesterásica sobre a butirilcolinesterase (BuChE), bem como a produção dos extratos metanólico, acetato de etila e hexânico extraídos a partir das folhas para a realização dos testes in vitro. Ainda, nessa mesma base de dados, foi encontrado um estudo de autoria de Kanazawa et al. (2003) relacionado à síntese de ácido barbacênico natural a partir da espécie Barbacenia flava. Na base de dados SciELO não foram obtidos resultados para os termos inseridos.

Inserindo-se os termos chave da tabela 3 na base de dados Science Direct, foram obtidos vinte e seis resultados para a pesquisa relacionados ao termo Barbacenia, mas depois da leitura dos artigos foram excluídos seis resultados, pois além de não fazerem menção aos termos de busca, em seus conteúdos 
constatou-se que o aparecimento desses artigos nos resultados de busca se deu porque a palavra-chave encontrava-se inserida em títulos de trabalhos utilizados na referência bibliográfica para a produção das pesquisas.

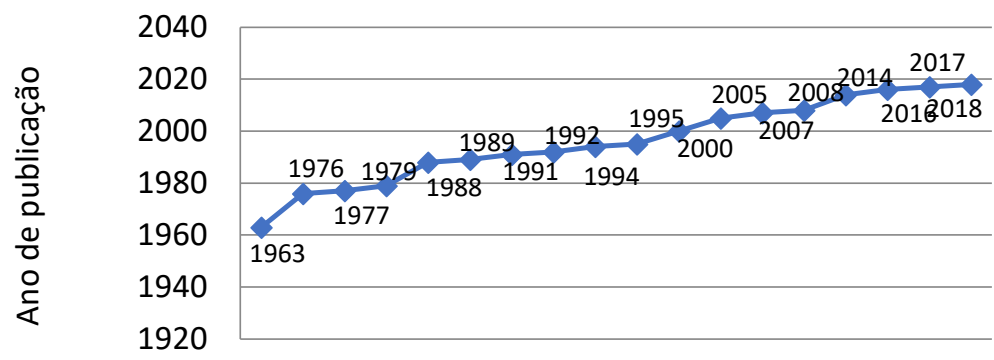

$\begin{array}{lllllllllllllllllll}1 & 1 & 1 & 1 & 2 & 1 & 2 & 0 & 2 & 0 & 0 & 1 & 1 & 1 & 1 & 2 & 1 & 2\end{array}$

Número de publicações

Gráfico1: Evolução do número de publicações sobre o gênero Barbacenia na plataforma Science Direct.

O gráfico 1 mostra o maior número de publicações ligadas à palavra-chave Barbacenia, relativa ao gênero, que também aparecia na designação relacionada para outras espécies pertencentes ao gênero, por isso, justifica-se a presença do termo Barbacenia nessa base de dados relatando-se o aparecimento de trabalhos relativos ao gênero e algumas espécies a partir do ano de 1963, onde o gênero é citado num estudo que trata da morfologia do pólen intitulado "Palinology" de autoria de Erdtman. Baker et al. (1976) desenvolveu um estudo que tratava do isolamento de dois triterpenos tetracíclicos extraídos de Barbacenia bicolor, já Sazima (1977) descreveu sobre a polinização feita pelo beija-flor na espécie Barbacenia flava e Pinto et al. (1988) publicou sobre a presença de bisnorditerpenos em Barbacenia flava.

Pant e Rastogi (1979) mencionam o gênero Barbacenia no trabalho intitulado "The Triterpenoids", um artigo de revisão que trata da identificação e distribuição de triterpenoides penta e tetracíclicos em plantas vasculares. Salatino et al. (1989) relata o gênero quando aborda sobre a presença de alcanos de ceras epicuticulares foliares de Velloziaceae, família que abrange o gênero Barbacenia, e também quando publica em 1991 um trabalho sobre a avaliação da plasticidade dos perfis alcanos de algumas espécies de Velloziaceae. Ainda em 1991, o gênero Barbacenia é mencionado no trabalho intitulado "The utility of leaf flavonoids as Taxonomic Markers in the subfamily and generic classification of the velloziaceae" de autoria de Williams et al. (1991), onde citavam o gênero Barbacenia como fonte de flavonoides.

Harborne e Williams (1994) publicaram uma pesquisa intitulada "Recent advances in the chemosystematics of the monocotyledons", a qual mencionava o gênero Barbacenia como sendo rico em misturas complexas abundantes em flavonoides e em outra pesquisa proposta também por Williams et al. (1994) intitulada "Differences in flavonoid patterns between genera within the velloziaceae" reafirmam o gênero como principais produtores desse tipo de molécula.

A pesquisa "The pollination biology of two species of Helicteres (Malvaceae) with different mechanisms of pollen deposition" proposta por Franceschinelli (2005) cita a comparação entre os mecanismos de polinização das espécies Barbacenia flava e de duas espécies do gênero Helicteres. Lüttge et al. (2007) publicaram um artigo intitulado "Physiological ecology of photosynthesis of five sympatric 
Prospeç̧ão científica e tecnológica: uso de nanopartículas de goma do cajueiro, avaliação das atividades anticolinesterásicas e antioxidantes da

species of Velloziaceae in the rupestrian fields of Serra do Cipó, Minas Gerais, Brazil", o qual mencionava sobre o estudo da ecologia fisiológica de cinco espécies brasileiras incluindo a espécie Barbacenia involucrata.

Um estudo publicado e intitulado "Flavonoids and anthocyanins from six Cassytha taxa (Lauraceae) as taxonomic markers" proposto por Murai et al. (2008) aponta a espécie Barbacenia conicostigma como rica em flavonoides glicosídeos e antocianinas. Outro artigo publicado por Hmeljevski et al. (2014) intitulado "Conservation assessment of an extremely restricted bromeliad highlights the need for population-based conservation on granitic inselbergs of the Brazilian Atlantic Forest" trata sobre a avaliação da conservação de uma bromélia restrita apenas em regiões de inselbergs graníticos e cita como exemplo a espécie Barbacenia fanniae como sendo também endêmica e restrita a esses ambientes.

Ainda na base de dados Science Direct, foi encontrado dois artigos publicados que mencionavam espécies pertencentes ao gênero Barbacenia. No primeiro artigo intitulado "Physiological responses to water deficit and changes in leaf cell wall composition as modulated by seasonality in the Brazilian resurrection plant Barbacenia purpurea" da autoria de Suguiyama et al. (2016) que trata das respostas fisiológicas em relação ao déficit hídrico e as mudanças na composição da parede celular foliada modulada pela sazonalidade na espécie Barbacenia purpurea.

No segundo artigo intitulado "Metabolic and growth responses of maize to successive drought and re-watering cycles" da autoria de Sun et al. (2016) abordam sobre as respostas metabólicas do crescimento do milho em ciclos sucessivos de seca e irrigação, onde o autor busca justificar que a diminuição dos açúcares no milho se deu através do uso dos carboidratos como fonte imediata de energia nas vias metabólicas dependentes de energia que são responsáveis pela reparação dos danos causados pela deficiência hídrica, sugerindo que o mesmo processo ocorre também na espécie Barbacenia purpurea.

Também foi encontrada uma publicação intitulada "The dual strategy of the bromeliad Pitcairnia burchellii Mez to cope with desiccation" da autoria de Vieira et al. (2017) onde o autor apresenta uma dupla estratégia para a espécie Pitcairnia burchellii Mez para lidar com a dessecação, abordando no corpo do artigo, sobre a capacidade da espécie Barbacenia purpurea tolerar também períodos relativamente curtos de dessecação, variando de poucos dias a poucas semanas.

Para o presente momento, foram encontradas duas publicações, sendo a primeira intitulada "Diversity of reserve carbohydrates in herbaceous species from Brazilian campo rupestre reveals similar functional traits to endure environmental stresses" da autoria de Joaquim et al. (2018) onde os autores apresentam estudos a respeito da diversidade de carboidratos de reserva em espécies herbáceas presentes no campo rupestre brasileiro onde revelam características funcionais similares para suportar estresses ambientais, parafraseando o gênero Barbacenia, e em especial a espécie Barbacenia plantaginea, como detentores dos maiores teores de carboidratos solúveis encontrados nas folhas, bainhas e raízes.

A segunda publicação trata-se de um artigo proposto por Mota et al. (2018) intitulado "Changes in species composition, vegetation structure, and life forms along an altitudinal gradient of rupestrian grasslands in south-eastern Brazil" onde os autores, dentre dez espécies estudadas, elegem também a 
espécie Barbacenia sp, localizada na Serra do Cipó na região sudeste do Brasil, como espécie de roseta com maior valor de importância.

Tabela 4: Número de artigos científicos publicados relacionados às palavras-chave.

\begin{tabular}{|l|c|}
\hline PALAVRAS-CHAVE & Web of Science \\
\hline Barbacenia* & 16 \\
\hline Barbacenia blanchetii* & 0 \\
\hline Barbacenia blanchetii AND nanothechology* & 0 \\
\hline Barbacenia blanchetii AND nanoparticle* & 0 \\
\hline Barbacenia blanchetii AND nanoparticle cashew gum* & 0 \\
\hline Barbacenia blanchetii AND essential oil* & 0 \\
\hline Barbacenia blanchetii AND aqueous extract* & 0 \\
\hline Barbacenia blanchetii AND hydroalcoholic extract* & 0 \\
\hline Barbacenia blanchetii AND alcohol extract* & 0 \\
\hline Barbacenia blanchetii AND ethanolic extract* & \\
\hline Barbacenia blanchetii AND ethyl acetate extract* & \\
\hline Barbacenia blanchetii AND hexane extract* & 0 \\
\hline Barbacenia blanchetii AND methanolic extract* & 0 \\
\hline Barbacenia blanchetii AND antioxidant activity* & \\
\hline Barbacenia blanchetii AND anticholinesterase activity* & 0 \\
\hline Barbacenia blanchetii AND Alzheimer's disease* & 0 \\
\hline
\end{tabular}

Na base de dados Web of Science, conforme a tabela 4, foram encontrados dezesseis artigos que citavam o gênero Barbacenia, apresentando trabalhos de cunho evolutivo, morfológico e filogenético em comparação a outras espécies vegetais. Ainda nessa mesma base de dados foram encontradas publicações relacionadas a outras espécies pertencentes ao gênero Barbacenia, como por exemplo, a espécie Barbacenia purpurea e a sua aplicabilidade no fornecimento de insights para o melhoramento agrícola de autoria de Suguiyama et al. (2014).

\section{CONCLUSÕES}

Os dados apresentados na presente prospecção científica e tecnológica mostraram que o uso da espécie vegetal Barbacenia blanchetii é praticamente inexistente, ou seja, trata-se de um novo campo de pesquisa a ser explorado, pois existe apenas um trabalho científico, de autoria de Barbosa et al. (2015) publicado, o qual menciona a respeito da eficácia, do experimento in vitro, da atividade do extrato metanólico sobre a butirilcolinesterase. Durante as pesquisas também não foi encontrado nenhum depósito em bancos de patentes, o que torna essa área bastante vasta e promissora para o desenvolvimento de pesquisas.

\section{REFERÊNCIAS}

BARBOSA, J. S.; ALMEIDA, V. M.; MARÇAL, R. M.; BRANCO, A.. RP-HPLC-DAD-MS(n) analysis and butyrylcholinesterase inhibitory activity of barbacenia blanchetii extracts. Natural Product Communications, v.10, n.6, p.983-986, 2015.

BAKER, P. M.; BARREIRO, E. L.; GILBERT, B. T..

Phytochemistry, v.15, n.5, 1976, p.785-787, 1975.

COSTA, E. V.; PINHEIRO, M. L. B.; MARQUES, F. A.; BRAGA, R. M.; SALES, M. B. H. L. N. First report of alkaloids in the genus Guatteriopsis (Annonaceae). Biochemical Systematics and
Ecology, v.37, p.43-45, 2009.

PAULA, R. C. M.; HEATLEY, F.; BUDD, P. M.. Characterisation of anacardium occidentale exudate polysaccharide. Polymer International, v.45, p.27-35, 1998.

DERMARIN, V.; ZAVOREO, I.; KES, V. B.; SIMUNDIC, A. M.. Biomarkers in Alzheimer's disease. Clinical Chemistry and Laboratory Medicine, v.49, p.773, 2011.

ELLMAN, G. L.. A new and rapid colorimetric determination 
Prospecção científica e tecnológica: uso de nanopartículas de goma do cajueiro, avaliação das atividades anticolinesterásicas e antioxidantes da espécie vegetal barbacenia blanchetii para aplicação na terapia da doença de alzheimer

of acetylcholinesterase activity. Biochem.Pharmacol., v.7, p.8895, 1961.

ERDTMAN, G.. Palynology. In: PRESTON, R. D.. Advances in botanical research. Leeds: Academic Press, 1963.

FEITOSA, C. M.. Plantas medicinais e a doença de Alzheimer. Campinas: Átomo, 2015.

FRANCESCHINELLI, E. V.. The pollination biology of two species of Helicteres (Malvaceae) with different mechanisms of pollen deposition. Flora: Morphology, Distribution, Functional Ecology of Plants, v.200, n.1, p.65-73, Apr, 2005.

HMELJEVSKI, K. V. Conservation assessment of an extremely restricted bromeliad highlights the need for populationbased conservation on granitic inselbergs of the Brazilian Atlantic Forest. Flora: Morphology, Distribution, Functional Ecology of Plants, v.209, n.5-6, p.250-259, 2014.

HARBORNE, J. B.; WILLIAMS, C. A.. Recent advances in the chemosystematics of the monocotyledons. Phytochemistry, v.37, n.1, p. 3-18, 1994.

HOLMES, C.. Dementia. Medicine, Baltimore, v.40, n.11, p.628-631, 2012.

JOAQUIM, E. O.. Diversity of reserve carbohydrates in herbaceous species from Brazilian campo rupestre reveals similar functional traits to endure environmental stresses. Flora, v.238, p. 201-209, 2018.

LÜTTGE, U.. Physiological ecology of photosynthesis of five sympatric species of Velloziaceae in the rupestrian fields of Serra do Cipó, Minas Gerais, Brazil. Flora: Morphology, Distribution, Functional Ecology of Plants, v.202, n.8, p.637646, 2007.

KONRATH, E. L.; PASSOS, S.; KLEIN-JÚNIOR, L. C.; HENRIQUES, A. T.. Alkaloids as a source of potential anticholinesterase inhibitors for the treatment of Alzheimer's disease. The Journal of Pharmacy and Pharmacology, v.65, n.12, p.1701-1725, 2013.

MAHDY, K.; SHAKER, O.; WAFAY, H.; NASSAR, Y.; HASSAN, H.; HUSSEIN, A.. Effect of some medicinal plant extracts on the oxidative stress status in Alzheimer's disease induced in rats. European Review for Medical and Pharmacological Sciences, v.16, n.3, p.31-42, 2012.

MOTA, G. S.. Changes in species composition, vegetation structure, and life forms along an altitudinal gradient of rupestrian grasslands in south-eastern Brazil. Flora, v.238, p.32-42, 2018.

MORZELLE, C. M.. Resíduos de romã (Punica granatum) na prevenção da doença de Alzheimer. Dissertação (Mestrado em Ciência e Tecnologia de Alimentos) - Universidade de São Paulo, Piracicaba, 2012.

MURAI, Y.; KOKUBUGATA, G.; YOKOTA, M.; KITAJIMA, J.; IWASHINA, T. Flavonoids and anthocyanins from six Cassytha taxa (Lauraceae) as taxonomic markers. Biochemical Systematics and Ecology, v.36, n.9, p.745-748, 2008.

MURRAY, A. P.; BELÉN, M. B.; CASTRO, M. J.; ALZA, N. P.; CAVALLARO, V.. Natural AChE inhibitors from plants and their contribution to Alzheimer's disease therapy. Current Neuropharmacology, v.11, n.4, p.388-413, 2013

OTAEGUI-ARRAZOLA, A.; AMIANO, P.; ELBUSTO, A.; URDANETA, E.; MARTÍNEZ-LAGE, P.. Diet, cognition and Alzheimer's disease: food for though. European Journal of Nutrition, Darmstadt, v.53, n.1, p.1-23, 2014.

PANT, P.; RASTOGI, R.. The triterpenoids. Phytochemistry, v.18, n.7, p. 1095-1108, 1979.

PEREIRA, D. M.; FERRERES, F.; OLIVEIRA, J. M.; GASPAR, L.; FARIA, J.; VALENTÃO, P.; SOTTOMAYOR, M.; ANDRADE, P. B.. Pharmacological effects of Cathatanthus roseus root alkaloids in acetylcholinesterase inhibition and cholinergic neurotransmission. Phytomedicine, v.17, n.8/9, p.646-652, 2010.

PINTO, A. C.; FRECHIANI, M. C.; PEREIRA, A. L.. Bisnorditerpenes with a barbacenane skeleton from Barbacenia flava. Phytochemistry, v.27, n.12, p.3917-3918, 1988.

POHANKA, M.. Alzheimer's disease and oxidative strees: a review. Current Medicinal Chemistry, v.21, n.3, p.356-364, 2014.

RAMIREZ-BERMUDEZ, J.. Alzheimer's disease: critical notes on the history of a medical concept. Archives of Medical Research, v.43, n.8, p.595-599, 2012.

RIEDEL, W. J.. Prevening cognitive decline in preclinical Alzheimer's disease. Current Opinion in Phamacology, v.14, p.18-22, 2014.

RAO, J. P.; GECKELER, K. E.. Polymer nanoparticles: preparation techniques and size-control parameters. Progress in Polymer Science, v.36, p. 887-913, 2011.

SALATINO, M. L. F.; SALATINO, A.; MENEZES, N. L.; MELLOSILVA, R.. Alkanes of foliar epicuticular waxes of Velloziaceae. Phytochemistry, v.28, n.4, p. 1105-1114, 1989.

SALATINO, A.; SALATINO, M. L. F.; MELLO-SILVA, R.; DUERHOLT-OLIVEIRA, I.. An appraisal of the plasticity of alkane profiles of some species of Velloziaceae. Biochemical Systematics and Ecology, v.19, n.3, p. 241-248, 1991.

SAZIMA. M.. Hummingbird Pollination of Barbacenia flava (Velloziaceae) in the Serra do Cipó, Minas Gerais, Brazil. Flora, v.166, n.3, p. 239-247, 1976.

SCOTTI, L.; MENDONÇA-JÚNIOR, F. J. B.; DA SILVA, M. S.; R. PITTA, I. R.; SCOTTI, M. T.. Biochemical changes evidenced in Alzheimer's disease: a mini-review. Letters in Drug Design \& Discovery, v.11, n.12, p.240-248, 2014.

SEIDL, C.. Pesquisa de substâncias naturais inibidoras da acetilcolinesterase. Dissertação (Mestrado em Ciências Farmacêuticas) - Universidade Federal do Paraná, Curitiba, 2010.

SINGH, M.; KAUR, M.; KUKREJA, H.; CHUGH, R.; SILAKARI, O.; SINGH, D.. Acetylcolinesterase inhibitors as Alzheimer therapy: from nerve toxins to neuroprotection. European Journal of Medicinal Chemistry, v.70, p.165-188, 2013.

SUGUIYAMA, V. F.. Physiological responses to water deficit 
and changes in leaf cell wall composition as modulated by seasonality in the Brazilian resurrection plant Barbacenia purpurea. South African Journal of Botany, v.105, p.270278, 2016.

SUN, C.; GAO, X.; CHEN, X.; FU, J.; ZHANG, Y. Metabolic and growth responses of maize to successive drought and rewatering cycles. Agricultural Water Management, v.172, p. 62-73, 2016.

TARAHOVSKY, Y. S.; KIM, Y. A.; YAGOLNIK, E. A.; MUZAFAROV, E. N.. Flavonoid-membrane interactions: Involvement of flavonoid metal complexes in raft signaling. Biochim Biophys Acta, 2014.
VIEIRA, E. A.. The dual strategy of the bromeliad Pitcairnia burchellii Mez to cope with desiccation. Environmental and Experimental Botany, v.143, p.135-148, 2017.

WILLIAMS, C. A.; HARBORNE, J. B.; GREENHAM, J.; EAGLES, J.. Differences in flavonoid patterns between genera within the Velloziaceae. Phytochemistry, v.36, n.4, p.931-940, 1994.

WILLIAMS, C. A.; HARBORNE, J. B.; MENEZES, N. L.. The utility of leaf flavonoids as Taxonomic Markers in the subfamily and generic classification of the Velloziaceae. Biochemical Systematics and Ecology, v.19, n. 6, p.483-495, 1991.

A CBPC - Companhia Brasileira de Produção Científica (CNPJ: 11.221.422/0001-03) detém os direitos materiais desta publicação. Os direitos referem-se à publicação do trabalho em qualquer parte do mundo, incluindo os direitos às renovações, expansões e disseminações da contribuição, bem como outros direitos subsidiários. Todos os trabalhos publicados eletronicamente poderão posteriormente ser publicados em coletâneas impressas sob coordenação da Sustenere Publishing, da Companhia Brasileira de Produção Científica e seus parceiros autorizados. Os (as) autores (as) preservam os direitos autorais, mas não têm permissão para a publicação da contribuição em outro meio, impresso ou digital, em português ou em tradução. 